\title{
Distribution and Characterization of Antibiotic Resistant Bacteria in the Sediment of Southern Basin of Lake Biwa
}

\author{
DAISUKe MiYAKE ${ }^{1}$, YASUHIRO KASAHARA ${ }^{2,3}$ and HiSAO MORISAKI ${ }^{1 *}$ \\ ${ }^{1}$ Faculty of Science and Engineering, Ritsumeikan University, 1-1-1 Noji-higashi, Kusatsu 525-8577, Japan \\ ${ }^{2}$ Graduate School of Biological Sciences, Nara Institute of Science and Technology, 8916-5 Takayama, Ikoma \\ 630-0101, Japan \\ ${ }^{3}$ Present address: Department of Bioresource Science, Ibaraki University, School of Agriculture, Ami, Ibaraki \\ 300-0393, Japan
}

(Received September 16, 2002-Accepted December 26, 2002)

The distribution of antibiotic-resistant bacteria in Lake Biwa sediment with depth from a relatively shallow $(0-1 \mathrm{~cm})$ to a relatively deep $(9-10 \mathrm{~cm})$ layer was investigated. More colonies formed on 100-fold diluted nutrient broth (DNB) agar medium than nutrient broth (NB) medium. The colony number and its difference between the two media decreased with depth. The resistance to antibiotics, ampicillin, chloramphenicol, erythromycin, rifampicin, streptomycin, sulfamethoxazole and tetracycline, was examined. The ratio of antibiotic-resistant strains increased with the depth of sediment. The isolates obtained from the deepest layer using antibiotic-free DNB medium included significantly more strains which were resistant to higher concentrations and many more kinds of antibiotics. In contrast, the isolates from the antibiotic-containing medium showed no such features. According to the $16 \mathrm{~S}$ rDNA sequence, the isolates from the shallowest layer belonged to the Actinobacteria phylum and the Alpha-, Beta- and Gamma-proteobacteria. The isolates from the deepest layer, however, belonged to the Firmicutes phylum and the Alphaproteobacteria with some exceptions. Many of the multi-resistant isolates were similar to the genus Ralstonia, Afipia, or Bacillus cereus, known for the biodegradation of xenobiotics or pathogenicities mediated via plasmids.

Key words: Lake Biwa, antibiotic resistance, Lake sediment, microbial flora, 16S rDNA sequence

The incidence of antibiotic-resistant bacteria in aquatic environments has increased dramatically. This increase is attributed to the selection of resistant bacteria and the ability of such bacteria to transfer plasmids encoding resistance ${ }^{3)}$.

There have been various studies concerning the distribution of antibiotic-resistant bacteria in the environment ${ }^{1,3,4,5,9)}$. These studies have compared the distribution of resistant bacteria among various places and identified the bacteria.

In the present study, we focused on a specific site rather than comparing various places and intended to study the distribution of the resistant bacteria along with a gradient of ecologically important factors. It has been reported that the

\footnotetext{
* Corresponding author; E-mail: morisaki@se.ritsumei.ac.jp, Tel: +81-77-561-2767, Fax: +81-77-561-2659
}

sediment of Lake Biwa is aerobic only at the thin uppermost layer $(2-3 \mathrm{~mm})$ and below this it becomes anaerobic ${ }^{20)}$. Thus, a great change in the concentration of dissolved oxygen with depth can be expected in the sediment. This observation is well consistent with our findings ${ }^{15)}$ that the activity for glucose mineralization by microbes decreases while the contribution of facultative anaerobes to the activity increases, with sediment depth. If one can obtain a profile of antibiotic-resistance concerning the sediment microbes having these features, it may provide a clue as to the mechanism for acquiring and distributing resistance to antibiotics in a natural environment as a function of environmental factors.

We report here that the profile of microflora resistant to antibiotics changed along with the depth of the sediment. The present study clarified the difference in microflora and 
characteristics of resistance between isolates from shallow and deep layers of sediment with respect to responses to antibiotic concentrations and multiplicity of resistance.

\section{Materials and Methods}

\section{Sampling of sediment}

Sediment samples were taken with a K-K core sampler (Hashimoto Science Cooperation, Kyoto, Japan) using a transparent acrylic tube $(5 \mathrm{~cm}$ in diameter) from a site $\left(35^{\circ} 00^{\prime} 39^{\prime \prime} \mathrm{N}, 135^{\circ} 52^{\prime} 30^{\prime \prime} \mathrm{E}\right.$, ca. $3 \mathrm{~m}$ deep) located in the southern basin of Lake Biwa. Samples were taken on June 24, 1996 and divided into four layers $(0-1,2-3,5-6$ and 9-10 cm deep from the boundary surface between the sediment and lake water) with a sterilized spatula. To avoid contamination from the tube wall, only the inner part of the sample was taken. Each of the divided samples was placed into a sterilized plastic bottle in a cooling box $\left(4^{\circ} \mathrm{C}\right)$ and brought back to our laboratory within several hours and kept in a refrigerator at $4^{\circ} \mathrm{C}$. The experiments were carried out within 2 days.

\section{Culture media and colony counts}

The samples from each depth were serially diluted in sterilized water $10^{2}-10^{4}$ fold. Then, nutrient broth (NB) or diluted nutrient broth (DNB) agar (1.5 wt\%) medium, each of which has been used to study microbes in various types of soils ${ }^{8}$, was poured on and mixed in with the samples. The NB medium contained $10 \mathrm{~g}$ of polypeptone (Japan Pharmaceuticals), $10 \mathrm{~g}$ of meat extract (Wako Pure Chemicals, Japan), and $5 \mathrm{~g}$ of $\mathrm{NaCl}$ in $1000 \mathrm{ml}$ of ion-exchanged water; $\mathrm{pH}$ 7.2. The DNB was a 100 -fold dilution of the NB medium. To examine the strains resistant to antibiotics, each of the following seven different antibiotics were added to the NB and DNB media at a final concentration of 70 $\mu \mathrm{g} / \mathrm{ml}$; sodium ampicillin, chloramphenicol, erythromycin, rifampicin, streptomycin sulfate, sulfamethoxazole and tetracycline hydrochloride, all purchased from Wako Pure Chemicals except rifampicin (Sigma, USA). The plates (three for each combination of sediment depth, dilution rate, kind of medium (NB or DNB) and kind of antibiotic including a blank) were incubated for 25 days at $20^{\circ} \mathrm{C}$ with the number of colonies counted everyday. The colonies were distinguished by marking the back of the Petri dish with of different colored pen and shape in each day. After the incubation, the strains were isolated randomly from the colonies (ca. 20 strains were isolated from either antibiotic-free or antibiotic-containing DNB medium for each layer of sediment) and examined as described below.

\section{Response to antibiotics}

The antibiotic resistance was studied with isolates from DNB plates, on which greater numbers of colonies were formed, by checking the growth of the isolate on DNB agar medium containing 35, 70 or $140 \mu \mathrm{g} / \mathrm{ml}$ of antibiotic. Only four antibiotics, ampicillin, chloramphenicol, streptomycin and sulfamethoxazole, were investigated because of the small number of isolates obtained from the medium containing other kinds of antibiotics. Isolates which could grow on the medium containing an antibiotic at a concentration higher than $70 \mu \mathrm{g} / \mathrm{ml}$, were judged to be resistant to the antibiotic in the present study. The number of antibiotics to which an isolate was resistant was counted to verify the multiplicity of resistance of the isolate.

\section{PCR amplification of $16 S \mathrm{rDNA}$}

After the characteristics of resistance had been examined, each isolate was cultured in DNB liquid medium and $1 \mathrm{ml}$ of the culture was frozen at $-40^{\circ} \mathrm{C}$ in an eppendorf tube. When DNA extraction by freezing and thawing alone was insufficient, lysozyme and phenol-chloroform-isoamylalcohol were used ${ }^{2,17)}$. The frozen bacterial cell suspension or the extracted DNA solution was pelleted by centrifugation after precipitation using $100 \%$ ethanol. The solution resuspended in TE buffer was used as a template for PCR amplification. A near-complete 16S rRNA gene (16S rDNA) was obtained from the extracted DNA by employing the primers 20F (5'-AGTTTGATCCTGGCTC-3') and 1510R (5'-GGCTACCTTGTTACGACT-3') in a PCR, corresponding to the conserved region of Escherichia coli in both primers. The PCR mixture consisted of 2.5 units of ExTaq (Takara Shuzo Co. Ltd, Japan), 1×Taq polymerase buffer, $200 \mu \mathrm{M}$ dNTPs, $100 \mathrm{pmol}$ of each primer and extracted DNA (100-200 ng) in $100 \mu \mathrm{l}$. The reaction mixture was overlaid with a drop of mineral oil and placed in a thermal cycler (Perkin Elmer, PJ2000). The cycling program was: 30 cycles of $95^{\circ} \mathrm{C}$ for $30 \mathrm{~s}, 60^{\circ} \mathrm{C}$ for $30 \mathrm{~s}$, and $72^{\circ} \mathrm{C}$ for $120 \mathrm{~s}$ and a final extension of one cycle of $72^{\circ} \mathrm{C}$ for $600 \mathrm{~s}$. The reaction mixture was separated by electrophoresis on $1 \%$ agarose gel and visualized with $0.1 \mu \mathrm{g} / \mathrm{ml}$ of ethidium bromide.

\section{DNA sequence and phylogenetic analysis}

The PCR-amplified 16S rDNA was purified with a QIA quick PCR purification kit (Qiagen, USA) and used as the template in sequencing reactions with the Thermo Sequenase dye terminator cycle sequencing pre-mix kit (Amersham Pharmacia, USA). Sequences were determined using the 
primers r1L (position 536-518 of E. coli $16 \mathrm{~S}$ rDNA), r2L (805-786), r3L (1111-1093), r4L (1406-1389), and M13RV. Products of the sequencing reactions were analyzed with an Applied Biosystems 373A sequencer (USA). Sequences were assembled using the software Auto Assembler version 2.1 (Applied Biosystems, USA), and analyzed with databases from the Ribosomal Database Project (http://rdp. life.uiuc.edu). For phylogenetic analysis of the sequence data sets, Clustal W version $1.8^{19)}$ was utilized and phylogenetic trees were constructed by the neighbor-joining method. The compared sequences spanned positions 335 to1405.

\section{Nucleotide sequence accession numbers}

All the sequences of $16 \mathrm{~S}$ rDNA determined in this study have been submitted to DDBJ under the accession number $\mathrm{AB} 074665$ to $\mathrm{AB} 074751$.

\section{Results and Discussion}

\section{Colony formation on antibiotic-free media}

The numbers of colonies that appeared on DNB agar medium increased stepwise with incubation time especially for the $0-1$ and $2-3 \mathrm{~cm}$ layers of the sediment as shown in Fig. 1. More colonies were observed in the shallower than deeper layers. More colonies appeared on DNB medium than NB medium as shown in Fig. 1 a) and b). It has been reported $^{7,8,13)}$ that isolates forming colonies in the later stages of incubation have lower growth rates than those forming colonies in the early stages. So, the sediment at $0-1$ and $2-3$ $\mathrm{cm}$ seems to contain many slow growing bacteria. On the
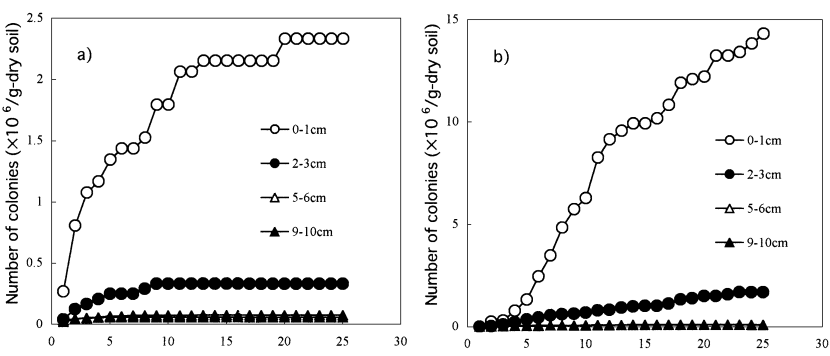

Incubation time (days)

Fig. 1. Number of colonies that appeared on NB (a) and DNB (b) agar medium with incubation time.

NB agar medium, most colonies (ca. $80 \%$ of total number) appeared within 10 days of incubation regardless of depth (Fig. 1 a).

The total number of colonies that formed during the entire incubation period ( 25 days) was greater on the DNB than NB medium. A similar difference has been observed in samples from grassland and a paddy field ${ }^{7,13}$. The difference in the total number of colonies between the DNB and NB medium decreased with depth. Oligotrophic bacteria, which can form colonies only on a dilute nutrient medium such as DNB, seem to be a relatively minor population in the deeper layers of sediment.

\section{Colony formation on antibiotic-containing media}

Very few colonies appeared on the media containing antibiotics compared to the antibiotic-free media, except in the NB medium containing sulfamethoxazole. The extent of decrease varied depending on the kind of antibiotic and

Table 1. Percentage of colonies on agar medium containing each antibiotic to that on antibiotic-free medium for the sediment samples from each depth.

\begin{tabular}{cccccccrc|cc}
\hline Medium & Depth of sediment $(\mathrm{cm})$ & ABPC & CP & EM & RFP & SM & SMX & TC & average & SD \\
\hline NB & $0-1$ & 2.3 & 0.2 & 0.8 & 0.1 & 2.3 & 14.5 & 0.6 & 1.05 & 1.00 \\
& $2-3$ & 7.1 & 0.0 & 3.2 & 1.3 & 6.9 & 98.8 & 1.7 & $3.37^{*}$ & 2.99 \\
& $5-6$ & 5.1 & 1.4 & 2.9 & 0.7 & 9.4 & 150.7 & 2.9 & $3.73^{*}$ & 3.17 \\
& $9-10$ & 2.0 & 2.0 & 3.0 & 0.0 & 17.8 & 83.7 & 4.4 & 4.87 & 6.50 \\
\hline \multirow{2}{*}{ DNB } & $0-1$ & 0.3 & 0.3 & 0.1 & 0.0 & 1.2 & 0.3 & 0.0 & 0.31 & 0.41 \\
& $2-3$ & 2.0 & 1.4 & 0.3 & 0.3 & 7.7 & 2.8 & 0.0 & $2.07^{*}$ & 2.68 \\
& $5-6$ & 7.0 & 2.0 & 0.0 & 0.3 & 8.2 & 15.8 & 1.2 & $4.93^{*}$ & 5.79 \\
& $9-10$ & 2.4 & 3.0 & 0.8 & 1.6 & 5.6 & 7.8 & 1.9 & $3.30^{*}$ & 2.50 \\
\hline
\end{tabular}

The upper half of the table corresponds to the isolates from NB and the lower half to those from DNB medium. The concentration of each antibiotic was $70 \mu \mathrm{g} / \mathrm{ml}$. The abbreviations used are as follows: ABPC (ampicillin), CP (chloramphenicol), EM (erythromycin), RFP (rifampicin), SM (streptomycin), SMX (sulfamethoxazole), and TC (tetracyclin).

* The average percentage of colonies on agar medium containing each antibiotic at this depth of sediment was significantly greater than the average value at the $0-1 \mathrm{~cm}$ layer. The data concerning sulfamethoxazole-containing NB medium was omitted from this calculation. 
depth of sediment as shown in Table 1.

The smallest number of colonies appeared on rifampicincontaining agar. The extent of the suppression was less for tetracycline, chloramphenicol and erythromycin and much less for ampicillin and streptomycin. When sulfamethoxazole was used, little suppression was observed and occasionally, in the NB medium, the number of colonies was greater than that on antibiotic-free medium. Brønstad et $a l .{ }^{5)}$ reported a similar result in a sandy agricultural soil (organic matter, 10\%): the percentage of isolates resistant to chloramphenicol or tetracycline was lowest and that resistant to sulfametoxazole was highest as we observed in the lake sediment.

The pattern of colony formation during the incubation showed some changes when an antibiotic was added. On the antibiotic-free DNB medium, ca. $1 / 3$ and $1 / 2$ of all colonies appeared during the later stage (between 13 to 25 days) of incubation in the samples from a depth of $0-1 \mathrm{~cm}$ and 2-3 $\mathrm{cm}$, respectively (see Fig. $1 \mathrm{~b}$ ). However, the number of colonies that appeared at a later stage on the antibioticcontaining DNB medium was far smaller (data not shown). The antibiotic-resistant strains may be mostly fast-growing bacteria.

It is noteworthy that the average percentage of antibioticresistant bacteria was significantly greater in the deeper than shallower layers of sediment (Table 1).

\section{Characteristics of the resistance}

The isolates from the lake-sediment were characterized with respect to response to the antibiotic concentration and multiplicity of resistance. To avoid a biased result from selecting strains from the medium on which only few colonies appeared, isolates randomly chosen from antibiotic-free and ampicillin-, chloramphenicol-, streptomycin- and sulfamethoxazole-containing DNB medium, where relatively greater numbers of colonies appeared, were used. The isolates from the shallowest $(0-1 \mathrm{~cm}$ in depth) and deepest $(9-10 \mathrm{~cm})$ layers of sediment were compared.

\section{a) The isolates from antibiotic-free DNB media}

First, the extent of the resistance of the isolates from the antibiotic-free media was examined by inoculating the strains on media containing an antibiotic at concentrations of 35,70 and $140 \mu \mathrm{g} / \mathrm{ml}$. An interesting feature was observed as shown in Fig. 2, i.e., the number of strains resistant to the higher concentration of antibiotics was greater in the isolates from the deep layer of sediment than the shallow layer, except the strains resistant to streptomycin: Here, the strains resistant to 70 or $140 \mu \mathrm{g} / \mathrm{ml}$ of an antibiotic were

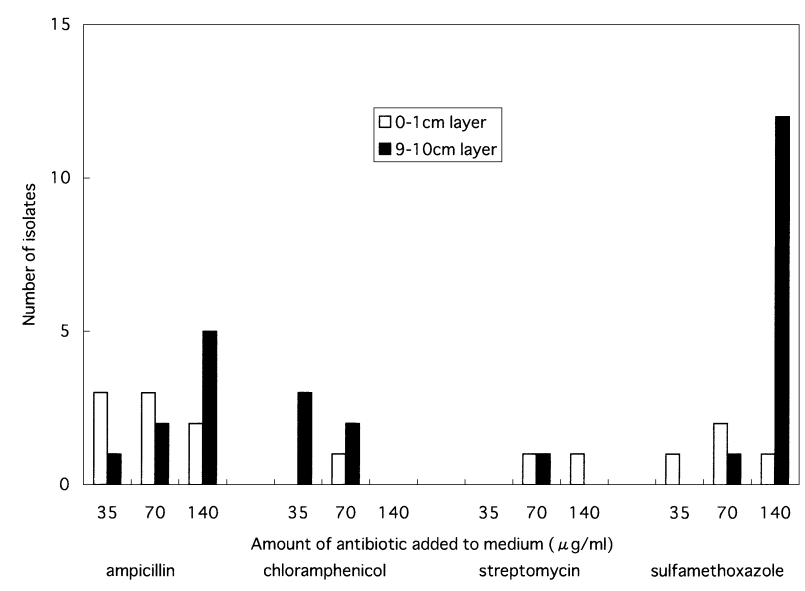

Fig. 2. Difference in number of isolates (from antibiotic free-media) resistant to various concentrations of antibiotics between the shallowest $(0-1 \mathrm{~cm})$ and deepest $(9-10 \mathrm{~cm})$ layers of sediment sampled.

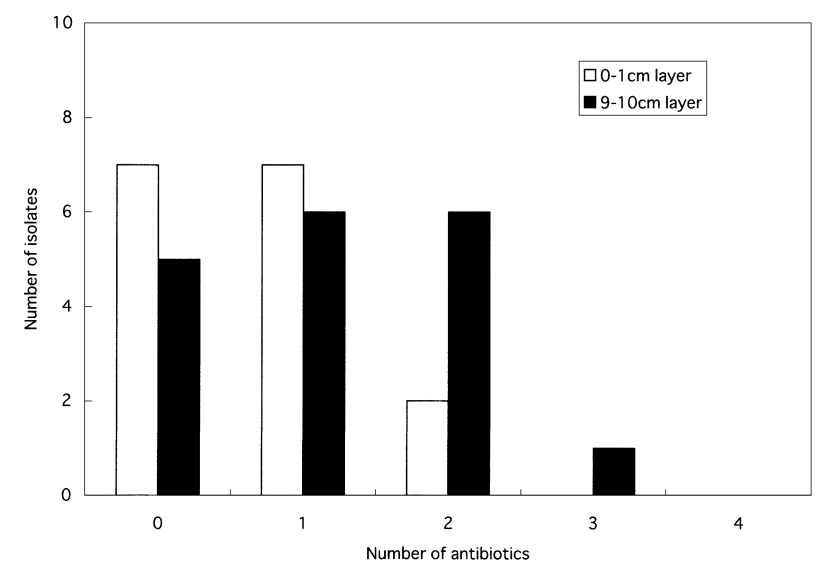

Fig. 3. Distribution of isolates (from antibiotic-free medium) resistant to no, single, and multiple antibiotics in the shallowest (0-1 $\mathrm{cm})$ and deepest $(9-10 \mathrm{~cm})$ layers of sediment sampled.

counted only once as resistant at the concentration to avoid double or triple counts at lower concentrations. The average to which the isolates from the deep layer of sediment were resistant was $109(\mathrm{SD}=44)$ and $134(\mathrm{SD}=19) \mu \mathrm{g} / \mathrm{ml}$ for ampicillin and sulfamethoxazole, respectively. These values were significantly greater $(\mathrm{t}$-test, $\mathrm{p}<0.05)$ than the average for the isolates from the shallow layer, $70(\mathrm{SD}=43)$ and 79 $(\mathrm{SD}=44) \mu \mathrm{g} / \mathrm{ml}$ for ampicillin and sulfamethoxazole, respectively: It must be pointed out that these differences may include some error, because, for example, a strain judged to be resistant to $140 \mu \mathrm{g} / \mathrm{ml}$ of an antibiotic may be resistant to a much higher concentration.

Furthermore, the isolates from the deep layer of sediment showed resistance to many more kinds of antibiotics (Fig. 
3). This value, $1.2(\mathrm{SD}=0.92)$ as an average, was significantly greater (t-test, $\mathrm{p}<0.05)$ than that of the isolates from the shallow layer, resistant to $0.69(\mathrm{SD}=0.70)$ kinds of antibiotics on average. As shown in Fig. 3, among the 18 isolates from the deep layer, 6 strains and 1 strain were resistant to 2 and 3 kinds of antibiotics, respectively. Contrary to this, among the 16 isolates from the shallow layer, only 2 strains were resistant to 2 kinds of antibiotics, and no strain was resistant to 3 or more kinds of antibiotics.

\section{b) The isolates from antibiotic-containing DNB media}

The isolates from the media containing each antibiotic showed no clear difference in the response to antibiotics as was observed in the isolates from antibiotic-free medium. However, a significant difference was observed among the isolates from ampicillin-containing medium. As shown in Fig. 4, the strains isolated from the shallow layer were significantly more resistant to the higher concentration of ampicillin ( $\mathrm{t}$-test, $\mathrm{p}<0.05$ ) than the strains from the deep layer. No such significant difference was observed between the isolates from the shallow and deep layer for the other antibiotics: The strains resistant to 70 or $140 \mu \mathrm{g} / \mathrm{ml}$ of antibiotic were counted only once to avoid double or triple counts at lower concentrations. In Fig. 4, some strains are resistant only at $35 \mu \mathrm{g} / \mathrm{ml}$, which appears to be lower than the concentration used for isolation $(70 \mu \mathrm{g} / \mathrm{ml})$. Here, it must be reiterated that the strains resistant to an antibiotic at $70 \mu \mathrm{g} / \mathrm{ml}$ were isolated and examined whether resistant to other antibiotics at 35,70 and $140 \mu \mathrm{g} / \mathrm{ml}$.

Concerning the number of antibiotics to which the isolates were resistant, no clear difference was observed

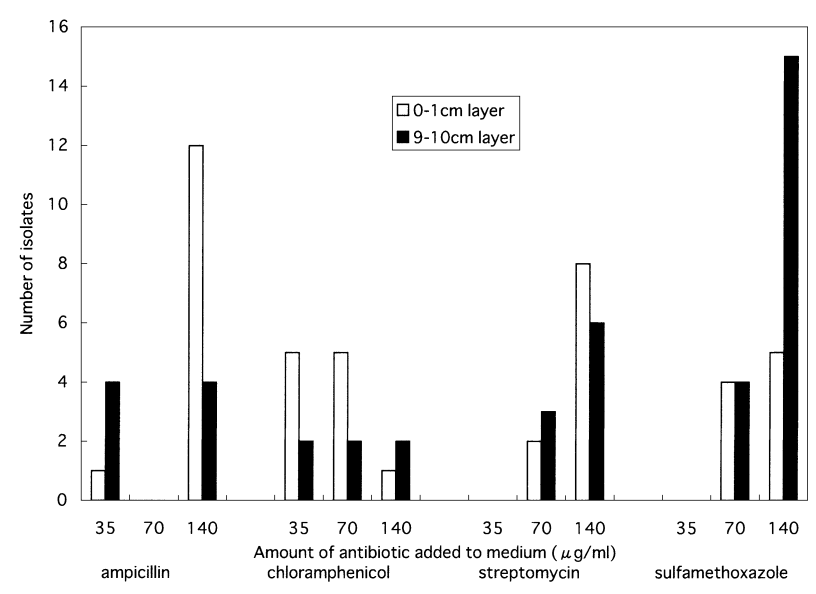

Fig. 4. Number of isolates (from antibiotic-containing media) resistant to various concentrations of antibiotics. The isolates from the shallowest $(0-1 \mathrm{~cm})$ and deepest $(9-10 \mathrm{~cm})$ layers of sediment sampled were compared.

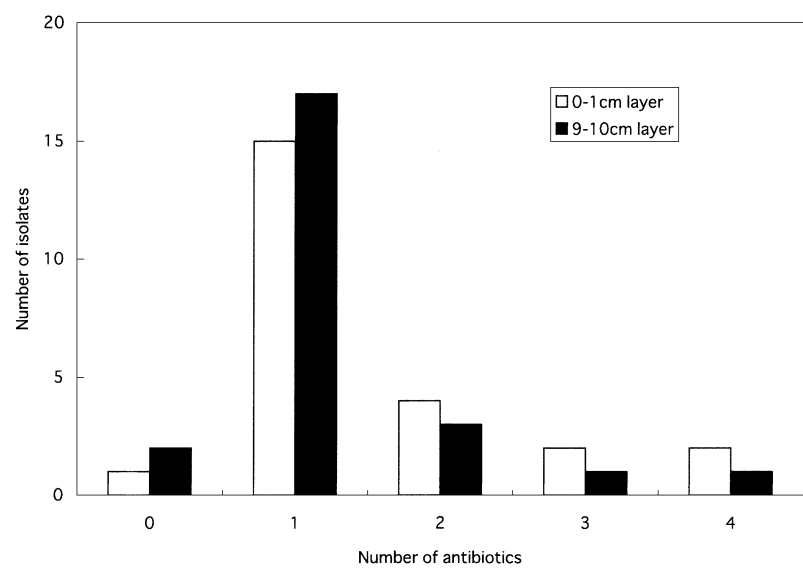

Fig. 5. Distribution of isolates resistant to no, single, and multiple antibiotics in the shallowest $(0-1 \mathrm{~cm})$ and deepest $(9-10 \mathrm{~cm})$ layers of sediment sampled.

Note that the isolates from medium containing each antibiotic at $70 \mu \mathrm{g} / \mathrm{ml}$ were examined.

between the isolates from the shallow and deep layers (Fig. 5). The isolates from the shallow layer were resistant to $1.5(\mathrm{SD}=1.0)$ kinds of antibiotics, not significantly different from the value, $1.3(\mathrm{SD}=0.85)$, of the isolates from the deep layer.

Only a few strains exhibited multiple-resistance to antibiotics in both the isolates from the deep and shallow layers of sediment as shown in Fig. 5. It has been reported that bacteria become more resistant to an antibiotic and even multi-resistant in places where the antibiotic is usually used via a mechanism such as the prevention of uptake, inactivation, and change in the target of the antibiotic ${ }^{14)}$. The isolates from the antibiotic-containing media did not show these features. Thus, selection pressure due to the presence of antibiotics is unlikely in the lake sediment.

\section{Characterization of resistant bacteria using the $16 \mathrm{~S} r$ DNA sequence}

The sequences of $16 \mathrm{~S}$ rDNA from 82 strains described in Fig. 3 and Fig. 5 were determined, resulting in the phylogenetic trees shown in Fig. 6 and 7 for the Gram-positive and negative isolates from both antibiotic-free and antibiotic-containing DNB medium: In these figures the other 5 isolates were added, although their response to antibiotic was not determined.

The Gram-positive isolates can be divided clearly into two groups as shown in Fig. 6. Many isolates from the shallow layer of sediment $(0-1 \mathrm{~cm})$ belonged to the Actinobacteria phylum. By contrast, almost all the isolates from the deep layer $(9-10 \mathrm{~cm})$ belonged to the Firmicutes phylum. 


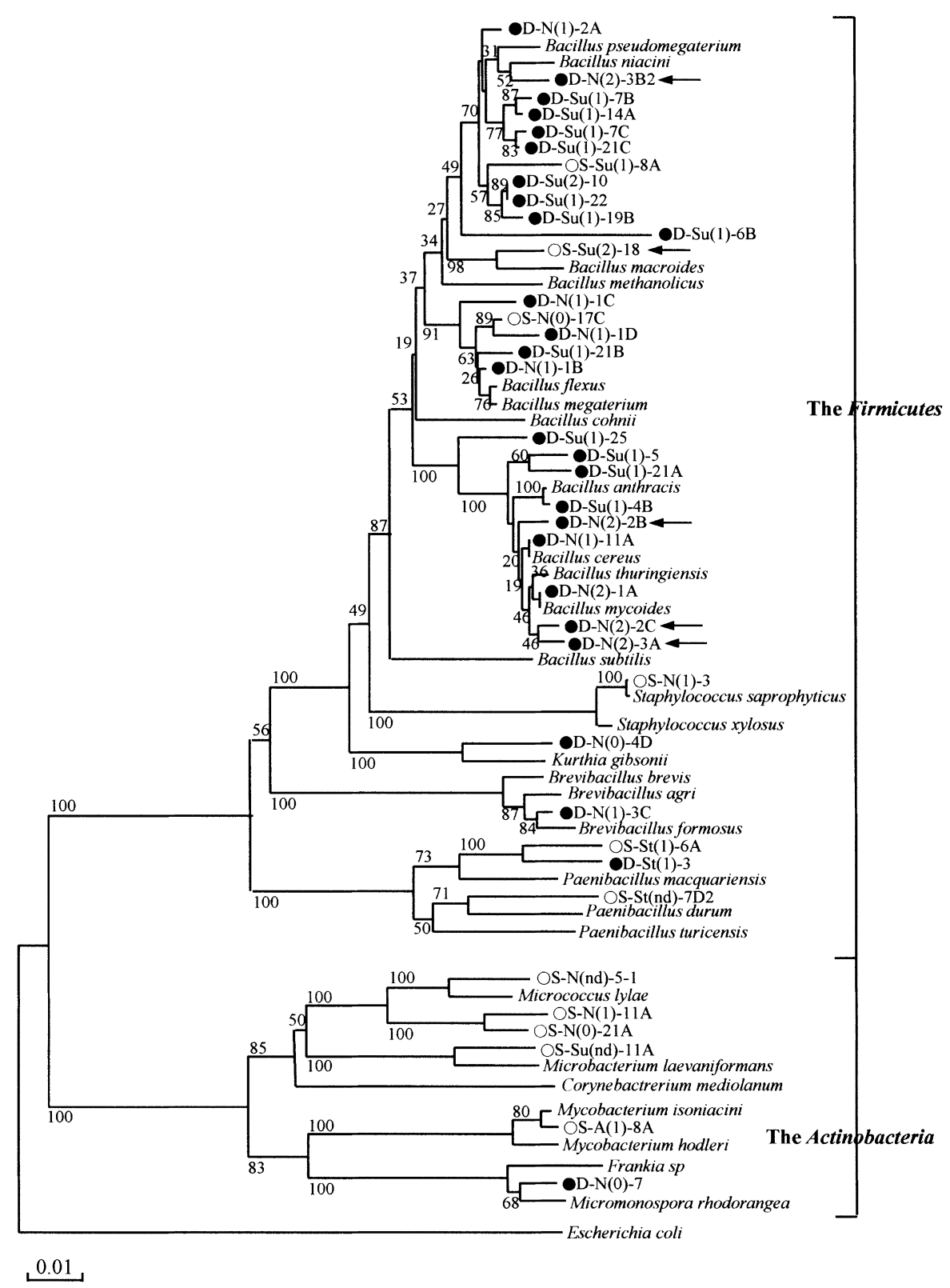

Fig. 6. Phylogenetic tree illustrating relationships of partial 16S rDNA sequences among Gram-positive isolates from the sediment of Lake Biwa and reference bacterial strains.

$\bigcirc$ indicates the strains isolated from the shallow layer of sediment and $\bigcirc$ indicates the strains from the deep layer of sediment. $\leftarrow$ indicates the strains resistant to more than two kinds of antibiotics. The tree was rooted with Escherichia coli as the outgroup. Numbers at nodes indicate bootstrap values out of 100 bootstrap re-samplings. The first part of the isolate's name indicates whether it was obtained from the shallow $(0-1 \mathrm{~cm}$ in depth) layer (S) or the deep $(9-10 \mathrm{~cm})$ layer $(\mathrm{D})$. The second part shows the kind of antibiotic contained in the agar medium used for isolation; ampicillin (A), chloramphenicol (C), streptomycin (St), sulfamethoxazole ( $\mathrm{Su}$ ) and no antibiotic $(\mathrm{N})$. The number in parentheses corresponds to the number of antibiotics to which the isolate was resistant except for some strains not determined (nd) yet. The last part shows the incubation time in days for the strain to form a visible colony on agar medium followed by an arrangement code.

The Gram-negative isolates also showed a clear difference between the shallow and deep layers of sediment as shown in Fig. 7. The isolates were packed together, according to depth, rather than scattered randomly. The isolates from the shallow layer distributed among the Alpha-, Betaand Gamma-proteobacteria, whereas the isolates from the deep layer were restricted to the Alphaproteobacteria with some exceptions. 


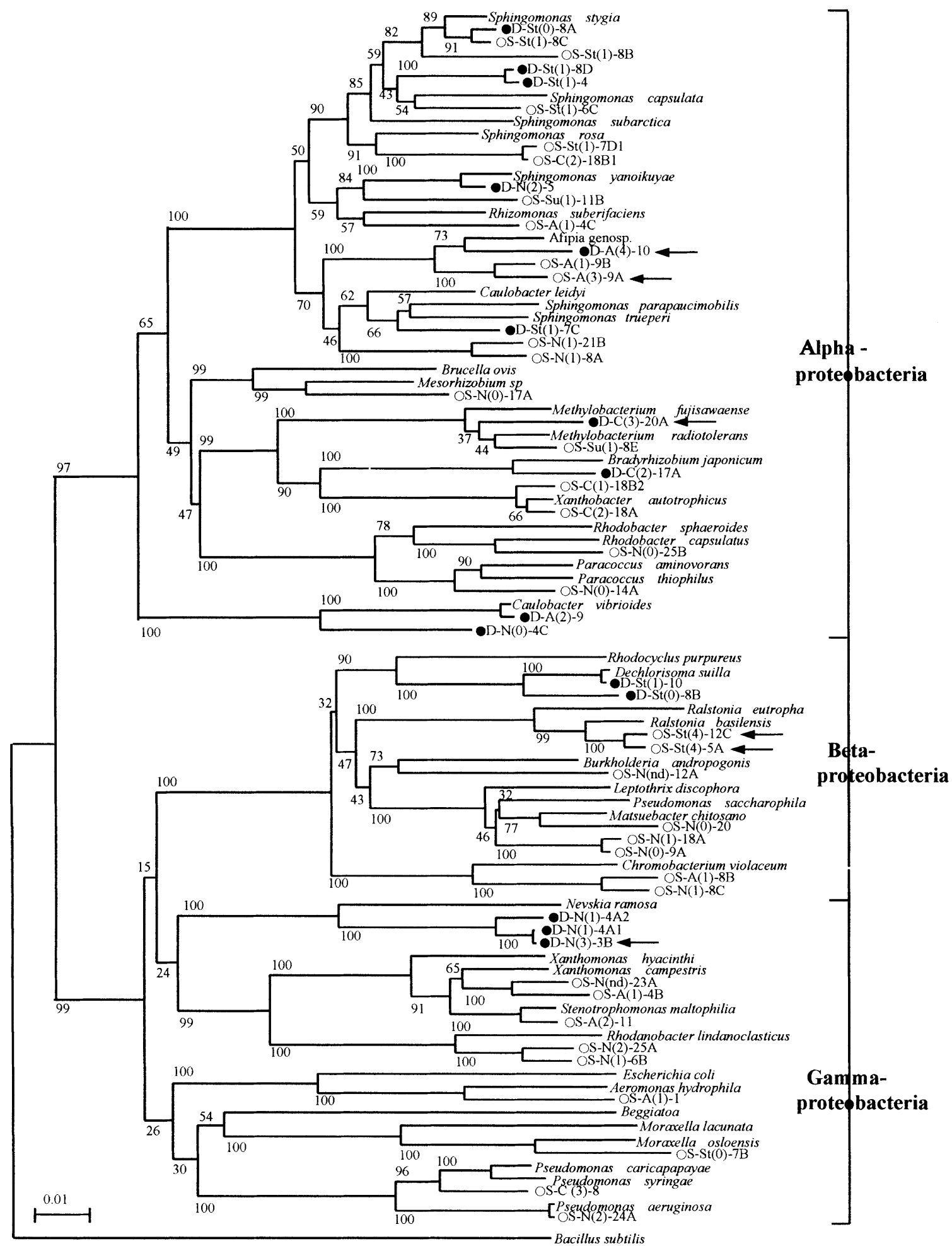

Fig. 7. Phylogenetic tree illustrating relationships of partial $16 \mathrm{~S}$ rDNA sequences among the Proteobacteria isolates from the sediment of Lake Biwa and reference bacterial strains.

$\bigcirc$ indicates the strains isolated from the shallow layer of sediment and $\bigcirc$ indicates the strains from the deep layer of sediment. $\leftarrow$ indicates the strains resistant to more than three kinds of antibiotics. The tree was rooted with Bacillus subtilis as the outgroup. Numbers at nodes indicate bootstrap values out of 100 bootstrap re-samplings. Each part of the isolate name was designated as in the legend of figure 6 . 
It is worth noting that the multiple-resistant isolates centered on specific strains as shown in Fig. 6 and 7. These strains are Ralstonia, Afipia and Bacillus anthracis, B. cereus, B. thuringiensis, and B. mycoides, known for their ability to degrade aromatic compounds or pathogenicity ${ }^{6,10,11,12,16,18)}$.

In the present study, the difference in the microflora of antibiotic-resistant bacteria between shallow and deep layers of lake sediment was clarified. The mechanism to acquire and/or transfer antibiotic-resistance among the microbes in sediment remains to be elucidated.

\section{Acknowledgements}

We thank Dr. K. Yokota (Lake Biwa Research Institute, Japan) for his help in sampling lake sediment. This work was supported in part by a Grant-in-Aid for Scientific Research (no. 14360203) from The Ministry of Education, Culture, Sports, Science and Technology of Japan.

\section{References}

1) Andersen, S.R. and R-A. Sandaa. 1994. Distribution of tetracycline resistance determinants among gram-negative bacteria isolated from polluted and unpolluted marine sediments. Appl. Environ. Microbiol. 60: 908-912.

2) Ausubel, F.M. et al. (eds.). 1990. Current Protocols in Molecular Biology, John Wiley \& Sons. New York, N.Y.

3) Baya, A.M., P.R. Brayton, V.L. Brown, D.J. Grimes, E.R. Cohen and R.R. Colwell. 1986. Coincident plasmids and antimicrobial resistance in marine bacteria isolated from polluted and unpolluted Atlantic ocean samples. Appl. Environ. Microbiol. 51: 1285-1292.

4) Belliveau, B.H., M.E. Starodub and J.T. Trevors. 1991. Occurrence of antibiotic and metal resistance and plasmids in Bacillus strains isolated from marine sediment. Can. J. Microbiol. 37: 513-520.

5) Brønstad, K., K. Drønen, L. Øvreås and V. Torsvik. 1996. Phenotypic diversity and antibiotic resistance in soil bacterial communities. J. Industrial Microbiol. 17: 253-259.

6) Dorte, B., S. Michael, H. Elke, F. Wittko and S. Frieder. 2000. Cometabolic degradation of dibenzofuran by biphenyl-cultivated Ralstonia sp. strain SBUG 290. Appl. Environ. Microbiol. 66: 4528-4531.

7) Golrach, K., R. Shingaki, H. Morisaki and T. Hattori. 1994. Construction of eco-collection of paddy field soil bacteria for population analysis. J. Gen. Appl. Microbiol. 40: 509-517.

8) Hattori, T. 1995. Analysis of Bacterial Communities in Soils: Eco-collection. T. Hattori (ed), ISK Series No. 8, Institute of Genetic Ecology, Tohoku University, Japan.

9) Hermansson, M., G.W. Jones and S. Kjelleberg. 1987. Frequency of anti-biotic and heavy metal resistance, pigmentation, and plasmids in bacteria of the marine air-water interface. Appl. Environ. Microbiol. 53: 2338-2342.

10) Itoh, K., R. Kanda, Y. Momoda, Y. Sumita, Y. Kamagata, K. Suyama and H. Yamamoto. 2000. Presence of 2,4-D-catabolizing bacteria in a Japanese arable soil that belong to BANA (Bradyrhizobium-Agromonas-Nitrobacter-Afipia) cluster in alpha-Proteobacteria. Microbes and Environments 15: 113-117.

11) Itoh, K., R. Kanda, Y. Sumita, H. Kim, Y. Kamagata, K. Suyama, H. Yamamoto, R.P. Hausinger and J.M. Tiedje. 2002. tfdA-like genes in 2,4-dichlorophenoxyacetic acid-degrading bacteria belonging to the Bradyrhizobium-Agromonas-Nitrobacter-Afipia cluster in alpha-Proteobacteria. Appl. Environ. Microbiol. 68: 3449-3454.

12) Logan, N.A. 1994. Bacterial Systematics. Blackwell Scientific Publications. Oxford, U.K.

13) Kasahara, Y. and T. Hattori. 1991. Analysis of bacterial populations in a grassland soil according to rates of development on solid media. FEMS Microbiol. Ecol. 86: 95-102.

14) Kerr, K.G. and R.W. Lacey. 1995. Why do we still get epidemics? p. 179-203. In P.A. Hunter, G.K. Darby and N.J. Russell (ed), Fifty years of antimicrobials: past perspectives and future trends. Cambridge University Press, Cambridge, U.K.

15) Okunishi, S., K. Yokota and H. Morisaki. 2000. Microflora and mineralization activity in the bottom sediment of Lake Biwa. Microbes and Environments 15: 1-10.

16) Perez-Pantoja, D., L. Guzman, M. Manzano, D.H. Pieper and B. Gonzalez. 2000. Role of tfdCIDIEIFI and tfdDIICIIEIIFII gene modules in catabolism of 3-chlorobenzoate by Ralstonia eutropha JMP134 (pJP4). Appl. Environ. Microbiol. 66: 1602 1608.

17) Sambrook, J., E.F. Fritsch and T.M. Maniatis. 1989. Molecular Cloning, Second ed., Cold Spring Harbor Laboratory Press. Cold Spring Harbor. N.Y.

18) Schneider, J., R.J. Grosser, K. Jayasimhulu, B. Kinkle and D. Warshawsky. 2000. Biodegradation intermediates of carbazole formed by Ralstonia sp. RJGII.123 isolated from hydrocarbon contaminated soil. Can. J. Microbiol. 46: 269-277.

19) Thompson, J.D., D.G. Higgins and T.J. Gibson. 1994. CLUSTAL W: improving the sensitivity of progressive multiple sequence alignment through sequence weighting, positions-specific gap penalties and weight matrix choice. Nucleic Acids Research 22: 4673-4680.

20) Yokota, K. 1996. Survey of the surface of lake sediment. Lake Biwa Research Institute Newsletters (A news brochure entitled "Oumia” in Japanese) 57: 1-2. 